\title{
LHCf: A LHC DETECTOR FOR ASTROPARTICLE PHYSICS*
}

\author{
RAFFAELlo D'Alessandro \\ AND \\ O. Adriani, L. Bonechi, M. Bongi, P. Papini \\ INFN and University of Firenze, Italy \\ G. CAstellini \\ INFN and IFAC CNR Firenze, Italy
}

A. Faus, J. Velasco

IFIC, Centro Mixto CSIC-UVEG, Valencia, Spain

M. Haguenauer

Ecole Polytechnique, Paris, France

Y. Itow, T. Mase, K. Masuda, Y. Matsubara, H. Matsumoto

H. Menjo, Y. Muraki, T. Sako, Y. Watanabe

STE Laboratory, Nagoya University, Japan

K. KASAHARA

Shibaura Institute of Technology, Saitama, Japan

T. TAmura, K. Yoshida

Kanagawa University, Yokohama, Japan

S. TORII

Waseda University, Japan

A. TRICOMI

INFN and University of Catania, Italy

W.C. TURner

LBNL, Berkeley, California, USA

(Received November 15, 2006)

A number of extremely high energy cosmic ray events have been observed by various collaborations. The existence of such events, above the Greisen-Zatsepin-Kuzmin (GZK) cut-off has to be explained by a top-down scenario involving exotic physics. Yet the results reported depend heavily on Monte Carlo procedures. The LHCf experiment will provide important data to calibrate the codes used in air shower simulations.

PACS numbers: 13.85.Tp, 13.85.Ni, 13.85.Qk

\footnotetext{
* Presented at the "Physics at LHC" Conference, Kraków, Poland, July 3-8, 2006.
} 


\section{Introduction}

A certain number of very interesting events were reported by the AGASA[1] collaboration and others $[2,3]$ in recent years. These events, whose spectra are shown in Fig. 1, are of extremely high energy and, if confirmed, must necessarily lead to a revision of our current understanding of particle physics.

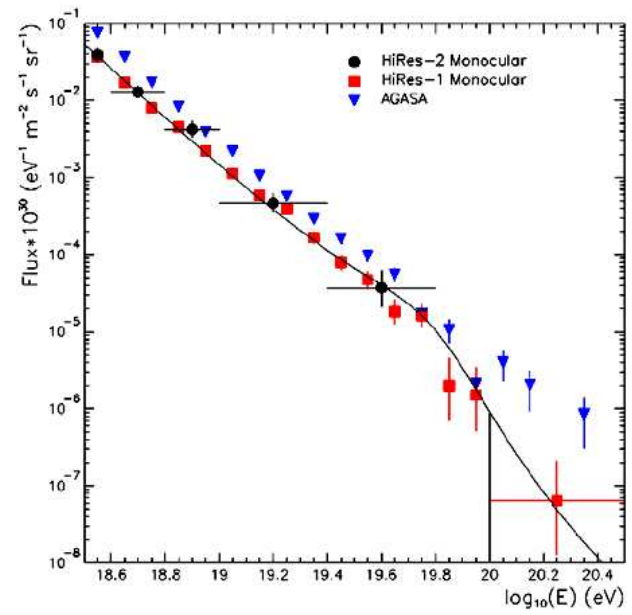

Fig. 1. Energy spectra of cosmic rays at very high energy. Triangles represent the AGASA experiment data, circles and squares the HiRes experiment data. A discrepancy between the two sets can be seen around $10^{20} \mathrm{eV}$.

Recently the Auger collaboration has shown some distributions of high energy cosmic rays, shown in Fig. 2, still the statistics are low and it is difficult to reach a definitive conclusion [2]. Yet the presence of these extremely high energy events poses some hard questions to our current knowledge of astroparticle physics. For one, the source of these particles cannot be extragalactic objects like Active Galactic Nuclei (AGN). Protons of this energy would never reach earth because of photo-nuclear interactions with the $2.7 \mathrm{~K}$ photons of the microwave background. The resonant interaction process (formation of $\Delta$ baryons) gives rise to an effective attenuation length of roughly 100 Megaparsecs. This is the GZK cut-off. Also other particles, like neutrons, nuclei, gammas have even lower attenuation length values. Thus one must invoke a top-down scenario where decays of cosmic strings or other exotic particles inside our galaxy, give rise to the extremely energetic decay products that are observed on the earth [5]. New answers on the existence of these cosmic rays will be given by a new generation of experiments like the new air shower detectors Auger [6] and TA [7] and eventually the EUSO [8] project which is under study. 
Yet observing the distributions in Fig. 2, one can glean how important it is that the energy scale used by the different collaborations is the correct one. The procedures for deriving the energy spectrum strongly depend on the nuclear interaction model used by the Monte Carlo codes that simulate the hadronic cascades in the atmosphere. In order to calibrate these codes and so choose an appropriate model it is very important to have a precise knowledge of the energy spectrum of forward emitted particles which are the main perpetrators of the air shower development.

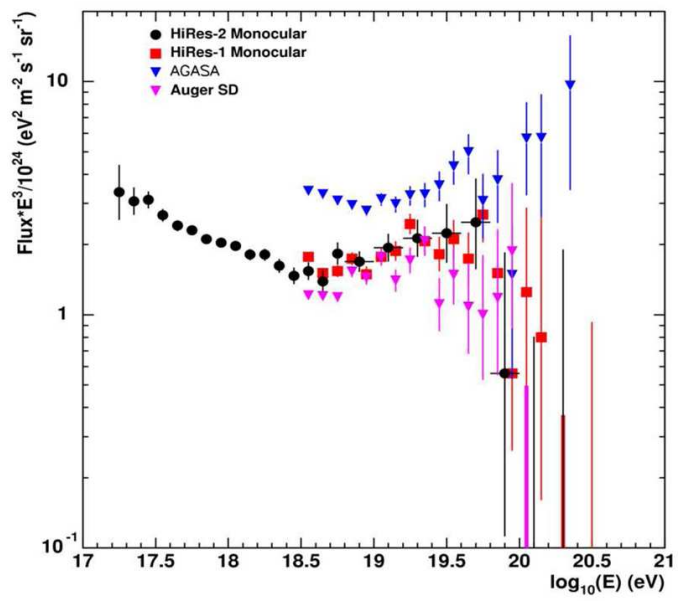

Fig. 2. The energy spectra of cosmic rays at very high energy obtained by the Auger experiment. A $20 \%$ reduction of the energy scale used by the AGASA group reduces significantly the discrepancy between data sets.

Another important puzzle concerning cosmic ray data, is the chemical composition of the rays themselves. In Fig. 3 we show data obtained by various collaborations around the "knee" region $\left(10^{16} \mathrm{eV}\right)$ on the shower maximum $X_{\mathrm{MAX}}[9]$. The position of the shower maximum clearly depends on the composition of the cosmic ray. A heavy nuclei will interact in the atmosphere before a same energy proton will. Thus a nucleus generated cascade will have shower maximum at a higher elevation than a proton one. Not being sure, however, which nuclear interaction model one should use, effectively reduces our ability to identify correctly the primary nucleus as can be seen by the Monte Carlo predictions superimposed on the data.

So again, we underline the importance of the correct choice of nuclear interaction model. Yet so far, the only high energy data set available against which one can calibrate the models, is still the one provided by the CERN UA7 collaboration [10] at $2 \times 10^{14} \mathrm{eV}$. The collaboration measured the energy distribution of photons and neutral pions in the rapidity range of $y=5-7$, 
thus providing a calibration point for the Monte Carlo codes up to an energy of $10^{14} \mathrm{eV}$. Above $10^{15} \mathrm{eV}$ the various model predictions begin to differ again due to the lack of experimental data checks.

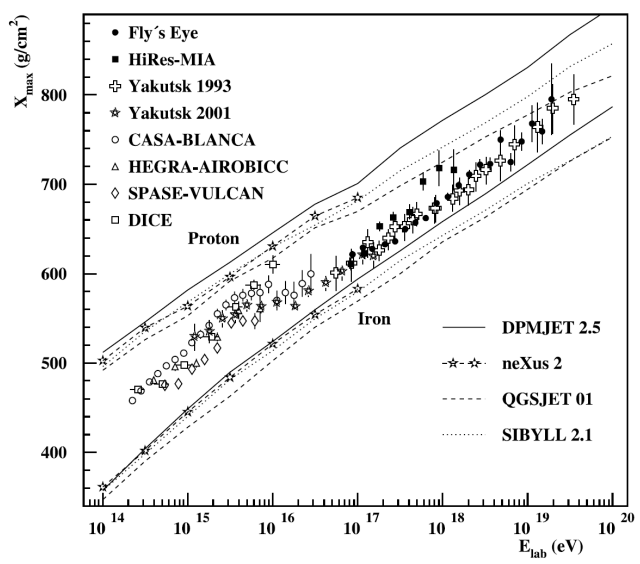

Fig. 3. The position of the shower maximum $X_{\mathrm{MAX}}$ is shown as a function of the primary cosmic ray energy. The various lines correspond to predictions made by different Monte Carlo codes.

The LHCf collaboration [11] plans to provide further data by using the LHC and its centre of mass energy of $14 \mathrm{TeV}$. This is an equivalent to a $2 \times 10^{17} \mathrm{eV}$ proton impacting in the atmosphere. The data points at this energy will allow us to discriminate between the various models used to predict shower development. In fact the most important quantities governing High Energy Cosmic Ray air shower development are:

1. the forward production spectra of photons and $\pi^{0}$ 's, 2 . the leading particle spectrum, 3. the total inelastic cross-section.

LHCf aims at providing information on the first two points, while the TOTEM experiment [12] will provide the third one.

\section{The LHCf experiment}

LHCf consists of two small electromagnetic calorimeters placed at \pm 140 meters from the interaction point (IP1) at the Large Hadron Collider (LHC) at CERN. The aim of the calorimeters is to measure down to pseudorapidity $y=\infty$ the energy spectra of photons and $\pi^{0}$.

The calorimeters are also capable of measuring accurately the shower position so as to reconstruct precisely the aperture angle of the two photons from $\pi^{0}$ decays. In Fig. 4 we show a schematic view of one of the calorimeters (ARM2) which consists of a sandwich of tungsten plates interleaved with scintillator tiles. 


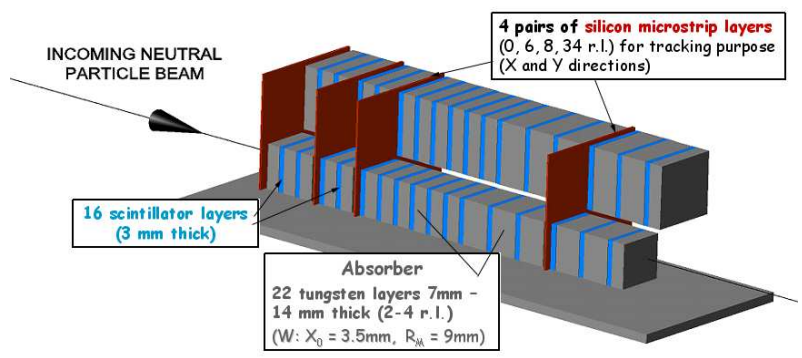

Fig. 4. A schematic drawing of the ARM2 LHCf calorimeter. The two towers have transverse dimensions of $25 \times 25 \mathrm{~mm}^{2}$ and $35 \times 35 \mathrm{~mm}^{2}$. The smaller one sits right in the middle of the LHC beam at $y=\infty$. The scintillator tiles are read out with phototubes while the silicon microstrip detectors have a pitch of 80 microns and provide a high precision measurement of the shower profile.

The shower position measurement is performed by four silicon micro-strip detectors. The peculiar geometry consisting of two separate mini towers, is dictated by the need of having minimum shower leakage from one tower to the adjacent one. In fact the silicon detectors are also capable of discriminating against this eventuality and are used to select very clean sample of events where only one photon has showered in one of the towers. Also by reconstructing the two separate showers from the $2 \gamma$ from $\pi^{0}$ decays LHCf obtains an excellent resolution on the invariant mass and thus has an excellent tool to calibrate the absolute energy scale. The calorimeters are capable of measuring photon energies up to a few $\mathrm{TeV}$. At these energies the charge released in the plastic scintillators corresponds to that of roughly $10^{4}-10^{5}$ MIPs. Great effort has been put in assuring a well behaved linear response of all the detector elements involved.

The tower sizes are comparable to the Moliere radius of the electromagnetic showers, thus there will be a certain amount of side leakage. This is corrected for by applying the position measurements of the silicon/scintillating fibre layers. Also the silicon detectors will provide very good discrimination against two photon events showering in the same tower which can introduce a systematic bias in the spectra measurement. In Fig. 5 there is a picture of the assembled prototype inside its casing.

The other calorimeter (ARM1) has a similar calorimeter structure (tungsten, scintillator sandwich) but the position measurement is achieved by four scintillating fibre layers. Also the overall geometry is slightly different, in as much the towers are rotated in order to facilitate the scintillating fibre extraction from the modules. Both calorimeters are housed in a beam absorbing structure called the TAN in which the two proton beams are steered in the two separate beam lines which circulate around the LHC machine. 


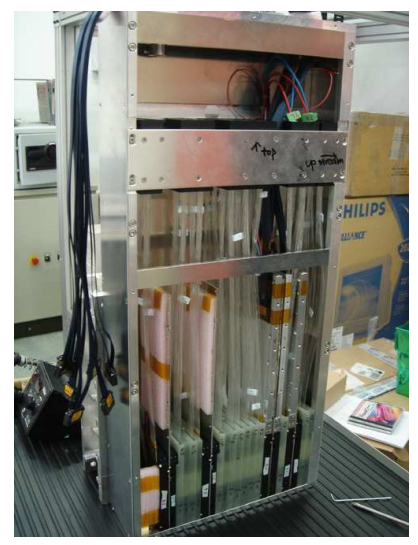

Fig. 5. Picture of the ARM2 LHCf calorimeter. The outside dimensions are $90 \mathrm{~mm}$ width, $300 \mathrm{~mm}$ length and $600 \mathrm{~mm}$ height. The light from the scintillator plates is guided by light fibres to the Hamamatsu photomultipliers. The black rectangular boxes house the silicon detector modules.

Thus the flux of charged particles is swept away and only the neutral ones reach the calorimeter surface. With two detectors we will be able to do systematics checks, different cuts on acceptance, eventually use a double arm trigger if backgrounds (beam gas, etc.) become too high. The acceptance of the two detectors is shown in Fig. 6.
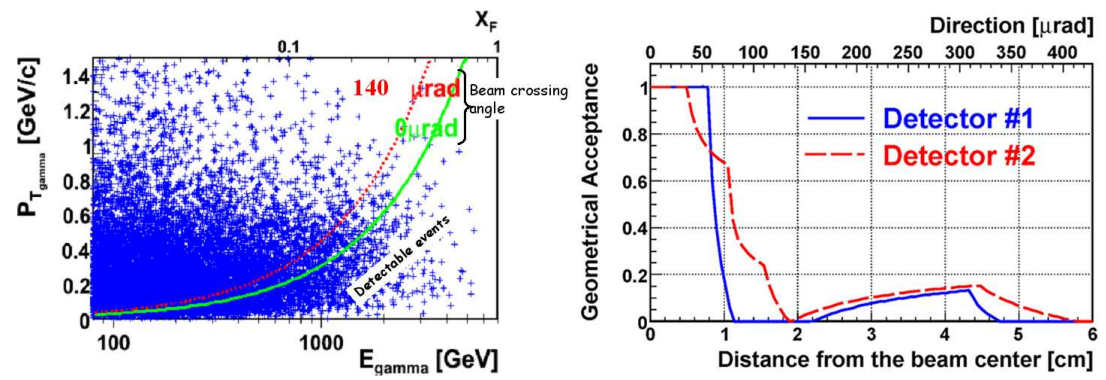

Fig. 6. $E_{\gamma}$ vs $P_{\mathrm{T} \gamma}$ correlation plot $\left(X_{\mathrm{F}}\right.$ is the Feynmann variable). The area below the curve is the one covered by the LHCf calorimeters. Also shown is the geometrical acceptance plot of the two arms for single $\gamma$.

\section{Expected performance and run scenario}

The expected shower profiles for photons and neutrons are shown in Fig. 7. Neutron energy spectrum can also aid in discriminating the various nuclear interaction models. If the neutron interacts within the first layers (one interaction length) we can measure its energy by fitting the longitudinal shower profile. 


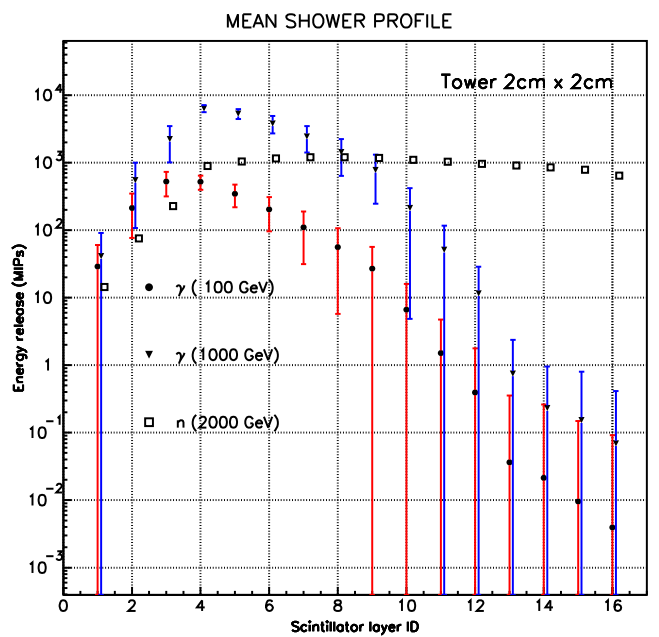

Fig. 7. Shower containment along the small calorimeter tower (Fluka simulated data). Full containment is obtained even for a $1 \mathrm{TeV} \gamma$. Also a $2 \mathrm{TeV}$ neutron shower profile is shown. In the latter case we can reconstruct the energy of the incident particle by fitting the shower profile.

A first test beam performed in 2004 with a very similar detector to the final one, gave very good energy resolution results (Fig. 8).

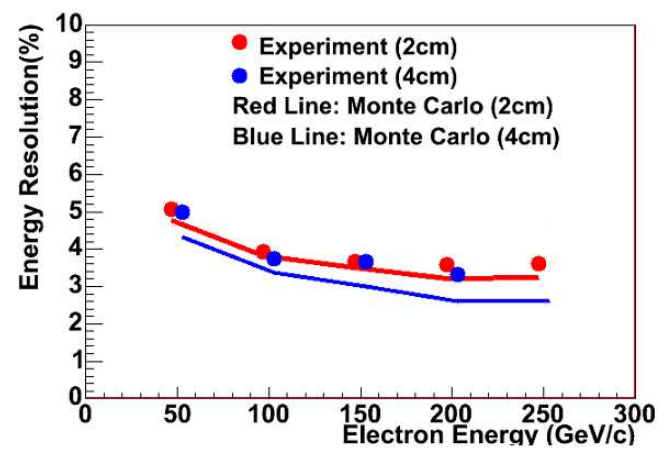

Fig. 8. Energy resolution as a function of incident article energy (electrons) for the $2 \times 2 \mathrm{~cm}^{2}$ tower and the $4 \times 4 \mathrm{~cm}^{2}$ one. The lines represent the corresponding Monte Carlo predictions.

Furthermore, the already good position resolution obtained with the scintillating fibres has now been bettered for the second calorimeter (ARM2) with the use of silicon microstrip detectors, whose shower transverse profile position resolution is shown in Fig. 9. The data refer to a Fluka simulation with up to $1.8 \mathrm{TeV}$ photons. 


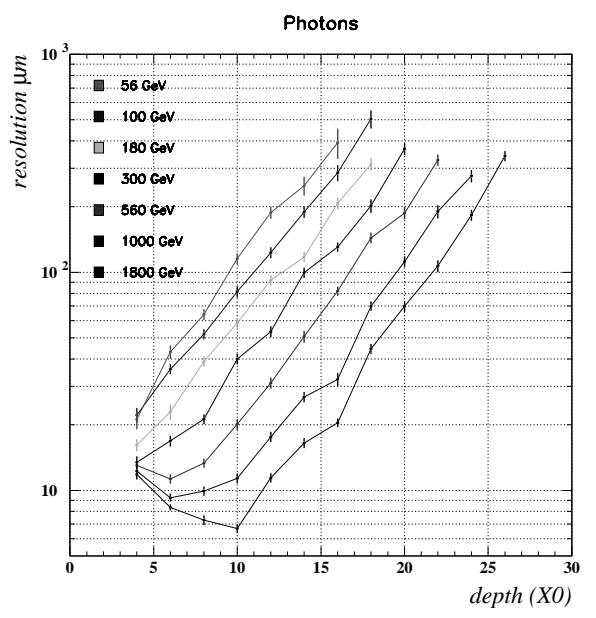

Fig. 9. Position resolution as a function of layer depth for the ARM2 calorimeter with silicon modules at various photon energies.

Also important is the very good double shower position resolution obtainable with this detector, which allows us to effectively separate two showers as close as $1 \mathrm{~mm}$ to each other. We have run extensive physics simulations so as to evaluate the different spectra obtainable by our apparatus at LHC. Depending on the nuclear interaction model used the energy spectra change more or less significantly. The models used are DPMJET3 [13], QGSJET-II [14] and SYBILL. A few results for photons and neutrons are shown in Fig. 10.
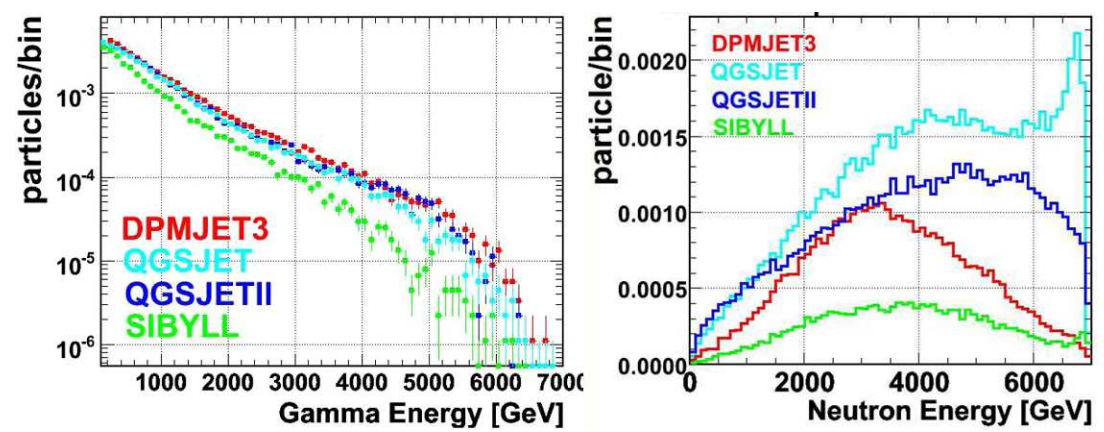

Fig. 10. Expected energy spectrum for $\gamma \mathrm{s}$ and neutrons according to different interaction models. The detector simulations used for these plots used the EPICS [15] code.

The discriminating power is already significant at $1 \mathrm{TeV}$ between SYBILL and the other codes, while discrimination between the other codes requires more statistics. 


\subsection{Run scenario}

The two calorimeters will be installed in LHC at the beginning of 2007 . Before then (August 2006) another test beam will have been performed at the CERN SPS accelerator. This test beam will validate the final detector. Once installed we foresee three operating phases:

- a first phase where we do parasitic running during the early stage of LHC commissioning (the end of 2007 or the beginning of 2008) after which we remove the detector when luminosity reaches $10^{30} \mathrm{~cm}^{-2} \mathrm{~s}^{-1}$ for radiation reason,

- a second phase where we re-install the detector at the next opportunity of a low luminosity run,

- a third phase where we run with heavy ions.

\subsection{Conclusions}

The LHCf apparatus will take data at LHC at the end of 2007. Great care has been taken in assuring a linear response of the detector and electronics up to the full $\mathrm{TeV}$ range of incoming photons. The calorimeter data will be used to calibrate air shower Monte Carlo codes up to the energy of $2 \times 10^{17} \mathrm{eV}$, thus providing invaluable input to questions posed since the first detection of extremely high energy cosmic rays. The two calorimeters are almost ready, and will be installed in the LHC tunnel at the beginning of 2007.

\section{REFERENCES}

[1] M. Takeda et al., Phys. Rev. Lett. 81, 1163 (1998).

[2] S. Yoshida, Rapporteur paper, 29th International Cosmic Ray Conference, Pune (2005).

[3] P. Sommers et al., Proc. of 29th International Cosmic Ray Conference, Pune (2005).

[4] D. Bergman et al., Proc. of 28th Int. Cosmic Ray Conf., Tsukuba (2003).

[5] S. Yoshida et al., Astrophys. J. 479, 547 (1997).

[6] J. Bluemer et al., Frontiers of Cosmic Ray Science, Universal Academy Press, 2003, p. 361.

[7] Y. Arai et al., Proc. of 28th International Cosmic Ray Conference, Tsukuba (2003).

[8] O. Catalano et al., Space Factory on International Space Station, Universal Academy Press, 2000, p. 21;

http://www.ifcai.pa.cnr.it/EUSO/docs/EUSOproposal.pdf 
[9] J. Knapp et al., Astropart. Phys. 19, 77 (2003).

[10] E. Pare et al., Phys. Lett. B242, 531 (1990).

[11] Technical Design Report, CERN-LHCC-2006-004, LHCF-TDR-001, February 7,2006

[12] Technical Design Report, CERN-LHCC-2004-002, TOTEM-TDR-001, January 7, 2004.

[13] S. Roesler et al., http://home.cern.ch/sroesler/dpmjet3.html

[14] S. Ostapchenko, hep-ph/0412332, hep-ph/0501093.

[15] See http://cosmos.n.kanagawa-u.ac.jp/ 